\title{
Temperature Dependent Soft X-ray Absorption Spectroscopy of Liquids
}

\author{
Jan Meibohm, ${ }^{1}$ Simon Schreck, ${ }^{1,2}$ and Philippe Wernet ${ }^{1, \text { a) }}$ \\ 1) Institute for Methods and Instrumentation for Synchrotron Radiation Research, \\ Helmholtz-Zentrum Berlin für Materialien und Energie GmbH, \\ Albert-Einstein-Strasse 15, 12489 Berlin, Germany \\ 2) Institut für Physik und Astronomie, Universität Potsdam, \\ Karl-Liebknecht-Strasse 24/25, 14476 Potsdam, Germany
}

A novel sample holder is introduced which allows for temperature dependent soft x-ray absorption spectroscopy of liquids in transmission mode. The setup is based on sample cells with x-ray transmissive silicon nitride windows. A cooling circuit allows for temperature regulation of the sample liquid between $-10^{\circ} \mathrm{C}$ and $+50^{\circ} \mathrm{C}$. The setup enables to record soft x-ray absorption spectra of liquids in transmission mode with a temperature resolution of $0.5 \mathrm{~K}$ and better. Reliability and reproducibility of the spectra are demonstrated by investigating the characteristic temperature-induced changes in the oxygen K-edge x-ray absorption spectrum of liquid water. These are compared to the corresponding changes in the oxygen K-edge spectra from x-ray Raman scattering.

a)wernet@helmholtz-berlin.de 


\section{INTRODUCTION}

The most important liquid on earth, water, is of particular scientific interest. Liquid water is involved in most chemical reactions and it exhibits more macroscopic anomalies than any other liquid ${ }^{1}$. Since the intermolecular forces in water are mainly due to hydrogen bonding, the complex microscopic structure of the hydrogen-bonding network is seen as the key for an understanding of the properties of water. Most of water's anomalies manifest themselves in peculiar macroscopic behavior under temperature change. Changes of its local structure are suspected to be responsible for these peculiarities and, therefore, experimental tools for temperature dependent investigations are needed to ultimately explain its macroscopic peculiarities.

Oxygen (O) K-edge x-ray absorption spectroscopy (XAS) has been established as a valuable probe of hydrogen bonding in liquid water ${ }^{2-10}$. XAS provides an element-, chemical siteand symmetry-specific probe of the unoccupied valence-electronic structure ${ }^{11,12}$. Therefore, temperature dependent XAS is expected to give valuable insight into the temperature effects in water.

Of the different methods to record x-ray absorption spectra (XA spectra) of light elements the most direct way is transmission mode XAS. In great difference to all other techniques, transmission mode XAS measures the absorption cross section directly via a primary process. It therefore provides high photon efficiency and leads to high-quality spectra. The most widely used alternative techniques of total fluorescence yield (TFY) and total electron electron yield (TEY) on the other hand detect the emission of the sample that arises from the decay of the core hole induced by the incident photon. The measurement of such secondary processes leads to conceptional problems such as, for TFY, low photon efficiency, self-absorption and saturation effects ${ }^{13,14}$.

Yet another way to record XA spectra of water is hard x-ray Raman scattering (XRS) ${ }^{3,12,15-17}$. This technique uses keV photons for inelastic scattering off the sample. The comparably large penetration depth of $\mathrm{keV}$ photons makes XRS an ideal tool to study water at high temperatures ${ }^{3,16}$, high pressures or a combination of both ${ }^{17-20}$. XRS spectra only agree with XAS in the so called dipole limit of small momentum transfer ${ }^{21}$. At an energy resolution

of $\leq 0.25 \mathrm{eV}$ as required for $\mathrm{O}$ K-edge spectroscopy of water the corresponding small cross section for XRS often implies comparably low signal-to-noise ratios compared to XAS. 
When applying XAS to liquid samples in general or to water in particular a major challenge is to combine the typically high vapor pressure of the liquid with the high absorbance of soft x-rays in matter. Techniques to overcome this challenge are based on the use of liquid jets in vacuum ${ }^{4,22-26}$ and cells with $\mathrm{x}$-ray transmissive membranes ${ }^{8,10,15,27-34}$. Liquid jets are used for TFY and electron-yield-based XAS. Currently, the only technique to perform transmission mode XAS on liquid samples is to use cells with a thickness below $1 \mu \mathrm{m}$. Yang and $\mathrm{Kirz}^{28}$ were the first to successfully implement transmission mode XAS on liquid water by enclosing the liquid in a thin cell with two x-ray transmissive silicon nitride $\left(\mathrm{Si}_{3} \mathrm{~N}_{4}\right)$ membranes, which then was placed in an evacuated chamber. This technique has been used and further developed since then for transmission mode XAS by various groups $8,15,27,29,30,35$. Such cells have also been applied recently to electron diffraction ${ }^{36}$. Temperature dependent transmission mode XAS however, has, to the best of our knowledge, not been reported yet. The question arises whether, based on the successful application of silicon-nitride based cells for temperature dependent IR studies in transmission mode ${ }^{37}$, such cells can also be used for temperature dependent transmission mode XAS.

We introduce here a novel silicon-nitride based sample holder for temperature dependent transmission mode XAS based on our previous design for transmission mode XAS described in Schreck et al. ${ }^{27}$. The high-quality XA spectra recorded with this setup allow for resolving small temperature effects in the O K-edge absorption spectra of liquid water. These are compared to the temperature effects in the recently published O K-edge spectra recorded with XRS.

\section{EXPERIMENTAL SETUP}

The setup described here represents an extension of the setup reported by Schreck et al. ${ }^{27}$. All details related to general instrumentation can be found there.

\section{A. Sample Holder}

The sample holder allows for temperature control of the liquid sample which is kept in a silicon-nitride based sample cell. The sample holder is depicted in detail in Figure 1 and consists of two separate parts that are shown in Figure 1(a) with the sample cell [yellow 
in Figure 1(a)] placed in between. The sample cell is briefly described in section II B. A photograph of the mounted sample holder is shown in Figure 1(b) with sample and cooling in- and outlets, one of the temperature sensors and the incident x-ray beam. The sample holder is made from copper to assure sufficient heat conductance. Figure 1(c) depicts several cuts through the 3D model of the sample holder. Two conical bores [see cut L-L in Figure 1(c)] clear the way to the membrane windows of the sample cell.

The novel temperature control of the sample cell is realized via a $4 \mathrm{~mm}$ cooling channel that goes through the sample holder [cut K-K in Figure 1(c)]. To transport the cooling liquid from the chiller outside the vacuum through liquid feedthroughs into the vacuum chamber and to the sample holder, two pipes are soldered directly to the sample holder. The cooling system allows a liquid flow of approximately $3 \mathrm{~L} / \mathrm{min}$. The comparably high flow enables a cooling time of approximately $2 \mathrm{~min} / \mathrm{K}$ at $23^{\circ} \mathrm{C}$ and $20 \mathrm{~min} / \mathrm{K}$ at $4{ }^{\circ} \mathrm{C}$. Once reached, the temperature of the sample holder and that of the sample has proven to be identical to within $\pm 0.25 \mathrm{~K}$ (see section I C).

\section{B. Sample Cell}

The sample cell is identical to the one described in Schreck et al. ${ }^{27}$. To summarize briefly, it consists of two lithographically-made silicon-based parts each with a $100 \mathrm{~nm}$-thick silicon nitride membrane at the center. In Figure 1(d) the upper part (left) and a cut view of the complete sample cell (right) are shown. Gold spacers create a $500 \mathrm{~nm}$ sample channel between the two silicon nitride membranes. The in- and outlet ports are connected to the vacuum pumps to allow for control of the sample thickness and to remove air bubbles that may have accumulated while mounting the sample ${ }^{27}$.

\section{Experimental Arrangement}

The sample holder is mounted on an xyz-manipulator in the center of a standard vacuum chamber $^{27}$. A pinhole can be placed in front of the sample holder to reduce the diameter of the incoming x-ray beam on the sample and thus, reduce effects of sample inhomogeneities, if needed. To detect the photon intensity a GaAsP diode (Hamamatsu, model G-112704) is moved into the x-ray beam behind the sample holder. The pinhole, the sample holder, 
Figure 1: (a) The two parts of the sample holder including the sample cell (yellow) sandwiched in between. (b) Photograph of the sample holder as it is mounted in the chamber. (c) 3D model of the sample holder shown with three cut views. (d) Left: 3D model of the upper part of the sample cell. Right: Cut view of the complete sample cell (taken from Schreck et al. ${ }^{27}$ ).

and the diode (each placed on separate xyz-manipulators) are aligned with respect to the incident x-ray beam. All components can separately be removed from the optical path.

The temperature sensor ["t-sensor", red arrow in Figure 1(b)] can be attached at the outside of the holder or directly at the sample cell. Since the temperature was found to be identical within the uncertainty of $\pm 0.25 \mathrm{~K}$ inside and at different positions on the outside surface of the sample holder it is assumed that the measured temperature is equal to that of the sample liquid. The blue and green arrows in Figure 1(b) illustrate the flow of the sample and the cooling liquid, respectively. The cooling liquid is flowed continuously, also during data acquisition to guarantee temperature stability to within $0.25 \mathrm{~K}$. In contrast, no flow of the sample liquid can be realized during measurement due to the small liquid channel cross sections. Instead, the sample can be slowly pushed back and forth to adjust the sample thickness and to remove air bubbles from the membrane windows if necessary. Radiation damage of the membranes or the sample as recently described ${ }^{38}$ are not observed for bulk liquids with our set up due to the comparably low photon density of the employed x-ray beams (see section IID). Thus, sample flow during measurement is not needed.

\section{Data Acquisition}

The data for this work were collected at the dipole beamline PM3 at the synchrotron light source BESSY II of Helmholtz-Zentrum Berlin in single bunch mode. Although the photon flux is much lower here than at an undulator beamline in multi-bunch mode it is high enough for measurements in transmission mode. At the same time, radiation damage ${ }^{38}$ is avoided. The beamline uses a plane grating monochromator with a 1221 lines/mm grating. The exit slit was set to $87 \mu \mathrm{m}$ and a $\mathrm{c}_{f f}$ value of 1.4 was used to minimize contributions of

higher-order light from the monochromator ${ }^{27,30}$. With these settings, the bandwidth of the 
incoming photons was $\Delta E \approx 0.25 \mathrm{eV}$ at $535 \mathrm{eV}$.

A transmission spectrum of the sample is obtained by dividing the diode current measured behind the sample cell filled with the sample liquid by that measured behind an empty sample cell. Using Lambert-Beer's law one obtains an absorption spectrum of the measured sample.

We present two types of $\mathrm{O}$ K-edge absorption spectra of liquid water here. For comparison of relative intensities of spectral features and for comparison with previous measurements we show spectra with relative intensities in arbitrary units. As the absorption measured in transmission depends on the sample thickness, these spectra need to be normalized in order to make spectra of different sample thicknesses and temperatures comparable. We found that our spectra do not depend considerably on whether normalized to the area between $530 \mathrm{eV}$ and $550 \mathrm{eV}$ (area normalization) or whether normalized to the edge-jump at $600 \mathrm{eV}$ (edge-jump normalization). Consistent with most previously published water O Kedge absorption spectra, we use area normalization for the absorption spectra with relative intensities in arbitrary units.

In addition we show $\mathrm{O}$ K-edge absorption spectra of liquid water displaying the (absolute) absorption cross section and its variation with temperature. The absorption cross section is independent of the sample thickness and the sample density, which makes it an appropriate quantity to extract temperature effects that are related to changes in the electronic structure of the sample. The absorption cross section was obtained from the measured transmission spectra by comparing one of the spectra in a given temperature series with the tabulated transmission far above the $\mathrm{O}$ K-edge $(\text { at } \approx 600 \mathrm{eV})^{39}$. In this way the sample thickness was determined and the absolute cross section could be calculated. As the absorption cross section of liquid water is independent of temperature at this energy far above the edge, all other spectra could be normalized to the same value there instead of determining the sample thickness for each spectrum separately (edge-jump normalization).

The $\mathrm{O}$ K-edge spectrum of liquid water exhibits the near edge x-ray absorption fine structure (NEXAFS) between $530 \mathrm{eV}$ and $550 \mathrm{eV}$. Its characteristic features include a preedge peak at $535 \mathrm{eV}$, a main-edge at about $538 \mathrm{eV}$ and a post-edge region between $540 \mathrm{eV}$ and $542 \mathrm{eV}$. Energy calibration of the XA spectra is done by setting the pre-edge peak in the $\mathrm{O}$ K-edge of liquid water at ambient temperatures $\left(23^{\circ} \mathrm{C}\right)$ to $535 \mathrm{eV}^{2}$. 
Figure 2: $\mathrm{O}$ K-edge x-ray absorption spectra of liquid water at 6 and $23^{\circ} \mathrm{C}$ measured in transmission mode. For each temperature two spectra measured on different sample cells are compared. In (a) the full measured photon energy rage is shown. In (b) only the NEXAFS region from 530 to $550 \mathrm{eV}$ is shown. Spectra are normalized to the area between $530 \mathrm{eV}$ and $550 \mathrm{eV}$ (area normalization).

\section{RESULTS}

In order to demonstrate reliability and quality of the spectra measured with the new setup, O K-edge XA spectra of liquid water at different temperatures are measured and analyzed. Because the spectrum of liquid water is very well known and the preparation of stable water samples is comparably easy, the O K-edge XA spectrum of liquid water is an adequate benchmark test for the new sample holder.

\section{A. Reproducibility and Quality of the Spectra}

In Figure $2 \mathrm{XA}$ spectra of liquid water at $6{ }^{\circ} \mathrm{C}$ and $23^{\circ} \mathrm{C}$ are shown, where for each temperature we compare two spectra measured on different sample cells. As can be seen,

the spectra match very well over the whole measured energy range [Figure 2(a)]. In the NEXAFS region which is shown in detail in Figure 2(b) the $6{ }^{\circ} \mathrm{C}$ spectra slightly deviate from one another in the post-edge region. These deviations determine the uncertainty of the spectra, which in general are measured on different sample thicknesses such as achieved on different days or during different experimental campaigns. In contrast we associate actual temperature effects to differences between spectra within a given temperature series i.e. measured during one day or directly after one another. As will be discussed in more detail in section IIIB the observed temperature effects are much larger than the measurement uncertainty. The spectra in Figure 2 demonstrate that it is meaningful to proceed now with the investigation of temperature changes as the spectra measured here with the new sample holder at room temperature agree well with previously published spectra ${ }^{27}$. 
Figure 3: Absorption cross section of liquid water around the O K-edge at 1.5, 23 and $39^{\circ} \mathrm{C}$ measured in transmission mode and the corresponding difference spectra with respect to $39^{\circ} \mathrm{C}$. In (a) and (b) the full measured photon-energy range is shown. In (c) and (d) only the NEXAFS region from 530 to $550 \mathrm{eV}$ is shown. Spectra are normalized to the edge-jump at $600 \mathrm{eV}$ (edge-jump normalization).

Figure 4: Absorption cross section of liquid water around the $\mathrm{O}$ K-edge at 4.0, 4.5 and $5.0^{\circ} \mathrm{C}$ measured in transmission mode and the corresponding difference spectra with respect to $4.5^{\circ} \mathrm{C}$. In (a) and (b) the full measured photon-energy range is shown. In (c) and (d) only the NEXAFS region from 530 to $550 \mathrm{eV}$ is shown. Spectra are normalized to the edge-jump at $600 \mathrm{eV}$ (edge-jump normalization).

\section{B. Temperature Series}

In order to study temperature effects in the O K-edge spectra of liquid water, series of spectra in different temperature ranges were measured. We found that the sample thickness changes when changing the temperature (it increases for increasing temperature). For large temperature changes by, typically, $40 \mathrm{~K}$ the sample thickness had to be adjusted to make sure the sample would not become too thick or too thin. This can be easily done in the presented set up by varying the He pressure in the chamber or by varying the pressure exerted by the vacuum pumps connected to the sample liquid ${ }^{27}$. For small temperature changes of $10-20 \mathrm{~K}$ the thickness did not have to be adjusted as Lambert-Beer's law was applicable and the spectra could be properly normalized to extract the temperature effects.

In the first temperature series, depicted in Figure 3, absorption cross section spectra for temperatures between $1.5^{\circ} \mathrm{C}$ and $39^{\circ} \mathrm{C}$ are shown in steps of $\approx 20^{\circ} \mathrm{C}$ as well as the corresponding difference spectra. As is shown in Figure 3, increasing temperature leads to an increase of the pre-edge peak while the main-edge is suppressed. At the same time, both the pre-edge peak and the main edge shift towards lower photon energies upon increasing temperature. This leads to two dips in the difference spectra at around $534.5 \mathrm{eV}$ and $536 \mathrm{eV}$. The post-edge region decreases with increasing temperature, which leads to a peak in the difference spectra around $542 \mathrm{eV}$. Temperature changes that can be reliably measured extend 
Figure 5: O K-edge x-ray absorption spectra of liquid water at temperatures of 1.5, 4.5, $8.8,16,23$ and $39^{\circ} \mathrm{C}$ measured in transmission mode. The black spectrum superimposed on each of the spectra is a copy of the $1.5^{\circ} \mathrm{C}$ spectrum. Spectra are normalized to the area between $530 \mathrm{eV}$ and $550 \mathrm{eV}$ (area normalization).

Figure 6: O K-edge x-ray absorption spectra of liquid water measured in transmission mode with the presented setup (XAS) [(a), top] and via hard x-ray Raman scattering (XRS) as recently published by Nilsson et al. ${ }^{12}$ [(a), bottom]. Spectra are normalized to the area between $530 \mathrm{eV}$ and $550 \mathrm{eV}$ (area normalization). Corresponding difference spectra are displayed in (b) for XAS and (c) for XRS. In (c) the dots and thin lines represent the connected points of the measured data and the thick solid lines represent the smoothened data.

up to $560 \mathrm{eV}$.

The second temperature series, shown in Figure 4, depicts absorption cross section spectra for temperatures between $4.0^{\circ} \mathrm{C}$ and $5.0^{\circ} \mathrm{C}$ in temperature steps of $0.5^{\circ} \mathrm{C}$. Even for such small temperature variations systematic spectral differences are observed. Moreover, these differences seem to be similar to those observed for the larger temperature steps: Increasing temperature leads to an increase of the pre-edge peak and a suppression of the main edge and the post-edge. Both pre- and main-edge peaks are shifted towards lower energies albeit with a smaller effect at $536 \mathrm{eV}$ compared to $534.5 \mathrm{eV}$ for the small temperature steps compared to the large ones. The observed systematic and consistent variation of the spectra for temperature steps of $0.5 \mathrm{~K}$ verifies that the set up reaches a temperature resolution of $0.5 \mathrm{~K}$ and better.

\section{COMPARISON TO PREVIOUS STUDIES}

The spectral changes in the $\mathrm{O}$ K-edge XA spectra upon temperature variation in liquid water measured here with XAS in transmission mode are consistent with the changes measured with $\mathrm{XRS}^{12,16}$. To substantiate this comparison we first show a stacked-plot presentation of our spectra in a similar way to the presentation in Pylkkänen et al. ${ }^{16}$. Second, 
difference spectra of certain temperatures are compared to XRS difference spectra from Nilsson et al. ${ }^{12}$.

In Figure 5 a stacked plot of liquid water XA spectra measured in transmission mode at temperatures between $1.5^{\circ} \mathrm{C}$ and $39^{\circ} \mathrm{C}$ is shown. In the given representation in Figure 5 the behavior of the pre-edge, main-edge and post-edge regions as described in section III B can be easily verified. The changes agree very well with the ones reported in Pylkkänen et $a l .{ }^{16}$. However, it is noted that in the XRS measurements of Pylkkänen et al. ${ }^{16}$ (spectra between $1.6^{\circ} \mathrm{C}$ and $43^{\circ} \mathrm{C}$ ) the main-edge enhances with increasing temperature in contrast to what is observed here. In combination with the decrease of the post edge with increasing temperature this results in an intersection of the $1.6^{\circ} \mathrm{C}$ spectra with each of the higher temperature spectra at around $539 \mathrm{eV}^{16}$. In the spectra presented here, this intersection occurs already at energies around $537 \mathrm{eV}$. The comparison in Figure 5 still demonstrates the high quality of the temperature dependent O K-edge XA spectra of water enabled by the novel setup. Compared to the corresponding state of the art XRS measurements by Pylkkänen et al. ${ }^{16}$, higher signal to noise ratios and higher resolutions can be achieved with a comparably simple set up at a comparably low soft x-ray flux of a dipole beamline in single-bunch mode. We note, though, that with XRS extreme temperatures and pressures can be reached in contrast to the presented approach ${ }^{17-20}$.

Figure 6(a) depicts our XA spectra (top) and XRS spectra from Nilsson et al. ${ }^{12}$ (bottom) for comparable temperatures. The respective difference spectra are shown in Figure 6(b) and (c). Qualitatively and as for the comparison to Pylkkänen et al. ${ }^{16}$, our transmission mode XA spectra and the the XRS spectra in Nilsson et al. ${ }^{12}$ exhibit similar temperature effects. However, marked differences are apparent when comparing the spectra quantitatively. We first note that the XA and XRS spectra in (a) are normalized to the same area and the respective differences are displayed on the same scale facilitating detailed comparison. The amplitudes in the $39^{\circ} \mathrm{C}-23^{\circ} \mathrm{C} \mathrm{XA}$ difference spectrum cannot be compared to the $60{ }^{\circ} \mathrm{C}-$ $22^{\circ} \mathrm{C}$ XRS difference spectrum due to the different temperature ranges. The shape of the curves, however, is very similar. We observe a large discrepancy when comparing the $4{ }^{\circ} \mathrm{C}-23{ }^{\circ} \mathrm{C}$ XA difference spectrum with the $4{ }^{\circ} \mathrm{C}-22^{\circ} \mathrm{C}$ XRS difference spectrum. Both the shape of the curves including relative peaks heights and the intersection of the spectra (root of the differences) and the magnitude of the effects are very different. While an intersection is found at $537 \mathrm{eV}$ in our transmission mode XAS data, it is located at $539 \mathrm{eV}$ 
in the XRS difference spectrum. Another root is located at $545 \mathrm{eV}$ for both the XA and the XRS difference spectra.

The observed discrepancies between the O K-edge XRS and XA difference spectra could be due to uncertainties related to the XRS spectra such as the removal of background (due to Compton scattering e.g.) and the influence of non-dipole contributions. The smoothing of the noisier XRS spectra in addition hampers more detailed comparison. Note though, that it cannot be fully ruled out that our transmission mode XA spectra suffer from small remaining inhomogeneity effects or the influence of remaining higher-order light contributions. Because of our careful preparation of the liquid samples and the consistency checks done in the first part, however, we do not believe that this can explain the observed differences. More detailed comparative measurements at the same temperatures and over wider temperature ranges could help understanding the discrepancies between the observed temperature effects in XA and XRS spectra.

\section{CONCLUSION AND OUTLOOK}

A novel setup for temperature dependent x-ray absorption spectroscopy of liquid samples for measurements in transmission mode is introduced. Its functionality and the reliability of the measured x-ray absorption spectra are shown for liquid water. Spectra were recorded at temperatures from $1.5^{\circ} \mathrm{C}$ to $39^{\circ} \mathrm{C}$. The high sensitivity to small spectral differences reached with this setup guarantees a temperature resolution of $\leq 0.5 \mathrm{~K}$ which is, to the best of our knowledge, unprecedented. The measured spectra exhibit characteristic behavior upon temperature variation. When temperature is increased, the pre-edge peak in the O K-edge x-ray absorption spectrum of liquid water increases whereas the main-edge decreases. At the same time, both pre-edge peak and main-edge shift towards lower photon energies for increasing temperature and the post-edge region is suppressed. This observed behavior is qualitatively consistent with that from recently published x-ray Raman scattering measurements. The recognized deviations between x-ray absorption spectroscopy and x-ray Raman scattering results could be due to remaining uncertainties of the different methods. The high quality of the transmission mode x-ray absorption spectra and the precise temperature control of the setup enable measuring temperature effects in steps of $0.5 \mathrm{~K}$ and below. In addition, the setup could allow for studying the temperature dependence of other bulk liquids or solvated 
species with x-ray absorption spectroscopy in transmission mode.

\section{ACKNOWLEDGEMENTS}

We are grateful to the BESSY II staff for continuous support and we thank in particular Christian Weniger.

\section{REFERENCES}

${ }^{1}$ M. F. Chaplin, "Water Structure and Science," (Oct 2013).

${ }^{2}$ S. Myneni, Y. Luo, L. Naslund, M. Cavalleri, L. Ojamae, H. Ogasawara, A. Pelmenschikov, P. Wernet, P. Vaterlein, C. Heske, Z. Hussain, L. Pettersson, and A. Nilsson, Journal of Physics-Condensed Matter 14, L213 (2002).

${ }^{3}$ P. Wernet, D. Nordlund, U. Bergmann, M. Cavalleri, M. Odelius, H. Ogasawara, L. Naslund, T. Hirsch, L. Ojamae, P. Glatzel, L. Pettersson, and A. Nilsson, Science 304, 995 (2004).

${ }^{4}$ J. D. Smith, C. D. Cappa, K. R. Wilson, B. M. Messer, R. C. Cohen, and R. J. Saykally, Science 306, 851 (2004).

${ }^{5}$ A. Nilsson, P. Wernet, D. Nordlund, U. Bergmann, M. Cavalleri, M. Odelius, H. Ogasawara, L.-Å. Näslund, T. K. Hirsch, L. Ojamäe, P. Glatzel, and L. G. M. Pettersson, Science 308, 793 (2005).

${ }^{6}$ J. D. Smith, C. D. Cappa, B. M. Messer, R. C. Cohen, and R. J. Saykally, Science 308, 793 (2005).

${ }^{7}$ K. Wilson, M. Cavalleri, B. Rude, R. Schaller, T. Catalano, A. Nilsson, R. Saykally, and L. Pettersson, Journal of Chemical Physical Chemistry B 109, 10194 (2005).

${ }^{8}$ N. Huse, H. Wen, D. Nordlund, E. Szilagyi, D. Daranciang, T. A. Miller, A. Nilsson, R. W. Schoenlein, and A. M. Lindenberg, Phys. Chem. Chem. Phys. 11, 3951 (2009).

${ }^{9}$ H. Wen, N. Huse, R. W. Schoenlein, and A. M. Lindenberg, Journal of Chemical Physics 131 (2009), 10.1063/1.3273204.

${ }^{10}$ A. Nilsson and L. G. M. Pettersson, Chemical Physics 389, 1 (2011).

${ }^{11}$ Joachim Stöhr, NEXAFS Spectroscopy (Springer, 1992). 
${ }^{12}$ A. Nilsson, D. Nordlund, I. Waluyo, N. Huang, H. Ogasawara, S. Kaya, U. Bergmann, L. A. Naeslund, H. Ostrom, P. Wernet, K. J. Andersson, T. Schiros, and L. G. M. Pettersson, Journal of Electron Spectroscopy and Related Phenomena 177, 99 (2010).

${ }^{13}$ S. Eisebitt, T. Böske, J.-E. Rubensson, and W. Eberhardt, Phys. Rev. B 47, 14103 (1993).

${ }^{14} \mathrm{P}$. Wernet, K. Kunnus, S. Schreck, W. Quevedo, R. Kurian, S. Techert, F. M. F. de Groot, M. Odelius, and A. Foehlisch, Journal of Chemical Physical Chemistry Letters 3, 3448 (2012).

${ }^{15}$ L.-Å. Näslund, J. Lüning, Y. Ufuktepe, H. Ogasawara, P. Wernet, U. Bergmann, L. G. M. Pettersson, and A. Nilsson, The Journal of Physical Chemistry B 109, 13835 (2005), pMID: 16852732.

${ }^{16}$ T. Pylkkänen, A. Sakko, M. Hakala, K. Hämäläinen, G. Monaco, and S. Huotari, Journal of Physical Chemistry B 115, 14544 (2011).

${ }^{17}$ P. Wernet, D. Testemale, J. Hazemann, R. Argoud, P. Glatzel, L. Pettersson, A. Nilsson, and U. Bergmann, Journal of Chemical Physics 123 (2005), 10.1063/1.2064867.

${ }^{18} \mathrm{H}$. Fukui, S. Huotari, D. Andrault, and T. Kawamoto, The Journal of Chemical Physics 127, 134502 (2007).

${ }^{19}$ Y. Cai, H. Mao, P. Chow, J. Tse, Y. Ma, S. Patchkovskii, J. Shu, V. Struzhkin, R. Hemley, H. Ishii, C. Chen, I. Jarrige, C. Chen, S. Shieh, E. Huang, and C. Kao, Physical Review Letters 94 (2005).

${ }^{20}$ W. L. Mao, H.-k. Mao, Y. Meng, P. J. Eng, M. Y. Hu, P. Chow, Y. Q. Cai, J. Shu, and R. J. Hemley, Science 314, 636 (2006).

${ }^{21}$ W. Schuelke, Electron Dynamics by Inelastic X-ray Scattering (Oxford University Press, 2007).

${ }^{22}$ M. Faubel, S. Schlemmer, and J. Toennies, Zeitschrift für Physik D Atoms, Molecules and Clusters 10, 2

${ }^{23}$ M. Faubel, B. Steiner, and J. Toennies, Journal of Chemical Physics 106, 9013 (1997).

${ }^{24}$ B. Winter, U. Hergenhahn, M. Faubel, O. Bjoerneholm, and I. V. Hertel, Journal of Chemical Physical 127 (2007).

${ }^{25}$ K. Kunnus, I. Rajkovic, S. Schreck, W. Quevedo, S. Eckert, M. Beye, E. Suljoti, C. Weniger, C. Kalus, S. Gruebel, M. Scholz, D. Nordlund, W. Zhang, R. W. Hartsock, K. J. Gaffney, W. F. Schlotter, J. J. Turner, B. Kennedy, F. Hennies, S. Techert, P. Wernet, and A. Foehlisch, Review of Scientific Instruments 83 (2012), 10.1063/1.4772685. 
${ }^{26}$ K. M. Lange and E. F. Aziz, Chemical Society Reviews 42, 6840 (2013).

${ }^{27}$ S. Schreck, G. Gavrila, C. Weniger, and P. Wernet, Review of Scientific Instruments 82 (2011).

${ }^{28}$ B. X. Yang and J. Kirz, Phys. Rev. B 36, 1361 (1987).

${ }^{29}$ P. Wernet, G. Gavrila, K. Godehusen, C. Weniger, E. Nibbering, T. Elsaesser, and W. Eberhardt, Applied Physics A 92, 511 (2008).

${ }^{30}$ M. Nagasaka, T. Hatsui, T. Horigome, Y. Hamamura, and N. Kosugi, Journal of Electron Spectroscopy and Related Phenomena 177, 130 (2010).

${ }^{31}$ J. Forsberg, L.-C. Duda, A. Olsson, T. Schmitt, J. Andersson, J. Nordgren, J. Hedberg, C. Leygraf, T. Aastrup, D. Wallinder, and J.-H. Guo, Review of Scientific Instruments 78 (2007).

${ }^{32}$ E. Aziz, A. Zimina, M. Freiwald, S. Eisebitt, and W. Eberhardt, Journal of Chemical Physics 124 (2006).

${ }^{33}$ M. Freiwald, S. Cramm, W. Eberhardt, and S. Eisebitt, Journal of Electron Spectroscopy and Related Phenomena 137, 413 (2004).

${ }^{34}$ D. K. Bora, P.-A. Glans, J. Pepper, Y.-S. Liu, and J.-H. Guo, The Review of Scientific Instruments 85, 043106 (2014).

${ }^{35}$ M. Nagasaka, H. Yzawa, T. Horigome, A. Hitchcock, and N. Kosugi, The Journal of Physical Chemistry C 117, 16343 (2013).

${ }^{36}$ C. Mueller, M. Harb, J. R. Dwyer, and R. J. D. Miller, The Journal of Physical Chemistry Letters 4, 2339 (2013).

${ }^{37}$ D. Kraemer, M. L. Cowan, A. Paarmann, N. Huse, E. T. J. Nibbering, T. Elsaesser, and R. J. D. Miller, Proceedings of the National Academy of Sciences 105, 437 (2008).

${ }^{38}$ O. Fuchs, F. Maier, L. Weinhardt, M. Weigand, M. Blum, M. Zharnikovc, J. Denlinger, M. Grunze, C. Heske, and E. Umbach, Nuclear Instruments \& Methods in Physics Research Section A-Accelerators Spectrometers Detectors and Associated Equipment 585, $172(2008)$

${ }^{39}$ B. L. Henke, E. M. Gullikson, and J. C. Davis, "X-ray interactions: photoabsorption, scattering, transmission, and reflection at $\mathrm{E}=50-30000 \mathrm{eV}, \mathrm{Z}=1-92$, atomic data and nuclear data tables," http://henke.lbl.gov/optical_constants/ (July 1993) 

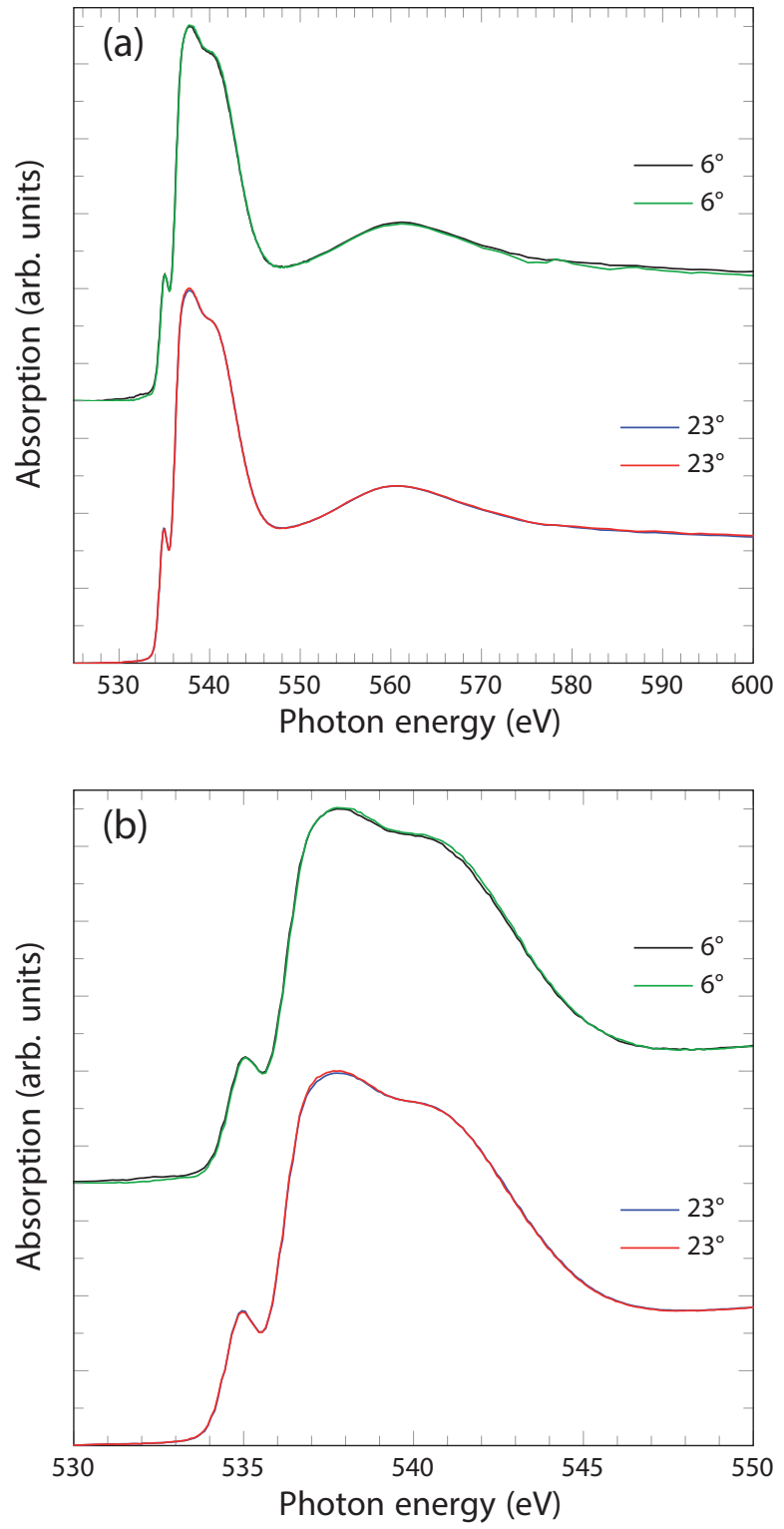

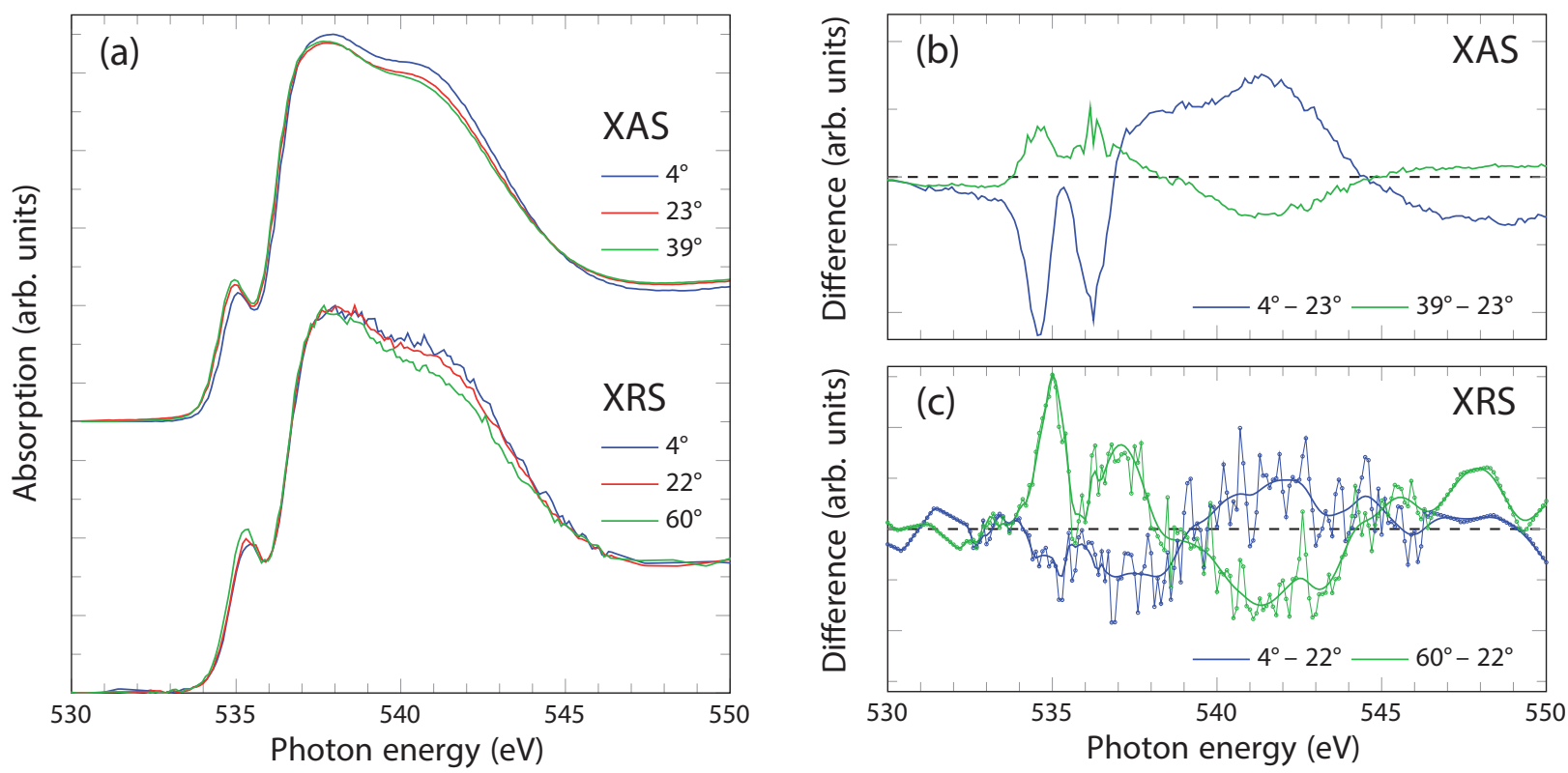